\title{
Constructing Resource Constrained Multicast Trees on WDM Networks with Limited Light-Splitting
}

\author{
Wen-Lin Yang, Chun-Tao Yang, and Yu-Chung Huang
}

\begin{abstract}
Traditionally, the optimization goal of multicast routing in WDM optical networks is to minimize the number of wavelengths and fibers used for the multicasting. In this paper, we consider a problem concerned about how to multicast several video streams simultaneously to a number of clients in a given WDM network with sparse light spitting, where the number of wavelengths on each link is given in advance. Our goal is to construct a number of multicast trees for all required streams so that the multiple constraints: bandwidth, delay and multicast capability, are satisfied and the total number of clients participating in all multicast sessions is also maximized. This problem is referred as profit-oriented and delay-constrained group multicast routing (PDGRM) problem. Two heuristic algorithms are proposed in this paper for this NP-hard problem. A set of experimental results are also provided to compare the performances of our heuristics with previous methods published in literature.
\end{abstract}

Index Terms-Group multicasting, light splitting, profit maximization, WDM networks.

\section{INTRODUCTION}

Recently, with the advance of communication technologies, many bandwidth hungry applications such as video-conferencing, video-on-demand (VOD), high definition television (HDTV), online games are believed to be the major driving forces behind the growth of e-commerce The network equipped with Wavelength Division Multiplexing (WDM) technology that can provide throughputs up to several terabytes through optical fibers is the most promising broadband platform [1]-[3]. Multicast routing mechanism can save large amount of bandwidths by sending messages with one packet to multiple destinations simultaneously. Hence, the multicast is a bandwidth saving method for transmitting multimedia streams.

To support multicast routing in the second generation of WDM networks, an optical node is required to be equipped with a light splitter which can split an input light signal into multiple outputs [4]. Such node is also called multicast capable (MC) node. Because of the high cost and complex architecture of MC nodes, only a part of optical nodes in a WDM network are permitted to have multicast capability [5]. Such optical networks are called WDM networks with sparse

Manuscript received June 15, 2015; revised September 16, 2015. This work was supported by a grant from the Ministry of Science and Technology, Taiwan, R.O.C. (MOST 103-2221-E-024-011).

W. L. Yang is with the Department of Computer Science and Information Engineering, National University of Tainan, Tainan, Taiwan (e-mail: wlyang@ mail.nutn.edu.tw).

C. T. Yang is with the Department of Electrical and Computer Engineering, National Chiao Tung University, Hsinchu, Taiwan.

David Huang is with the Department of Electrical Engineering, National University of Tainan, Tainan, Taiwan. light splitting. For a multicast implementation on a WDM network with sparse light splitting, more than one multicast trees may be required for the same multicast session. It is because of the lack of MC nodes to support light splitting. A multicast tree established on a WDM network is usually called a light-tree. Hence, it is very common to have a light forest to implement a multicast application on a WDM with sparse splitting. Different wavelengths are then needed and allocated to different light-trees in a light forest to support multiple concurrent transmissions. Many studies [5]-[7] about wavelength assignments for multicast trees have been proposed. Their goal is to minimize the number of required wavelengths subject to the multicast capability constraint.

In this paper, we are interested in the problem which is concerning about how to fully utilize the wavelength resources on the wavelength division multiplexing (WDM) optical networks when many clients send out requests simultaneously to receive the same or distinct programs from a video server built on the network. Obviously, we need multiple multicast trees to carry all the required video streams. For the owners and operators of the WDM networks, they may have interests in whether there is a method to accommodate all these multicast trees on the resource constrained WDM optical networks so that the number of serviced clients are maximized.

On the contrast to previous studies on WDM networks, a number of constraints about multicast capability of nodes, the number of wavelengths on a link (the bandwidth constraint), the multicast delay bound and the number of serviced clients are jointly considered in this study for a given WDM network with sparse light splitting. To the best of our knowledge, this is the first work about the cross-layer design consideration at application layer for the multicast on WDM networks.

Our goal is to construct a number of multicast trees for all required streams without violating the aforementioned constraints so that the total number of clients participating in multicasting is maximized. This problem is referred as the profit-oriented and delay-constrained group multicast routing (PDGRM) problem in this study. The group multicast routing problem is a well-known NP-hard problem [8], [9]. Two heuristics based on greedy and k-shortest paths strategies are proposed in this paper for the PDGRMproblem.

\section{PROBlem Formulation AND ASSUMPTIONS}

As shown in Fig. 1, a WDM network can be represented by an acyclic direct graph $G(V, E)$, where $V$ is set of optical nodes and $E$ is a set of directed links. Each link $l \in \mathrm{E}$ is associated with a bandwidth resource $\mathrm{w}(l)$ and a transmission delay $\rho(l)$. The $\mathrm{w}(l)$ represents the number of wavelengths can be allocated to different concurrent transmissions on the link $l$. A special node $\underline{\underline{S}}$ is served as a 
server node which is able to transmit a number of video programs simultaneously. Hence, we assume that only outgoing links are incident from $S$ to represent streaming direction.

At each optical switch node $u \in \mathrm{V}(u \neq S)$, it provides communication service to a LAN (local area networks) where a number of clients are attached. The clients covered by each non-server node may send requests to subscribe the same or distinct video streams provided by the server node $S$. An optical switch node $u \in \mathrm{V}$ is defined to be a destination if at least one clients at $\mathrm{u}$ send out subscription requests. That is, request $(u)>0$. A destination set $D$ is then defined as follows: $\mathrm{D}=\{u \mid$ request $(u)>0, u \in \mathrm{V}\}$.

Let $M$ denote the set of video programs subscribed by clients and required to be transmitted concurrently. Our goal is to construct m multicast trees: $G T=\left\{T_{0}, \cdots, T_{m-1}\right\}$, where $m$ is the number of elements in $M$.

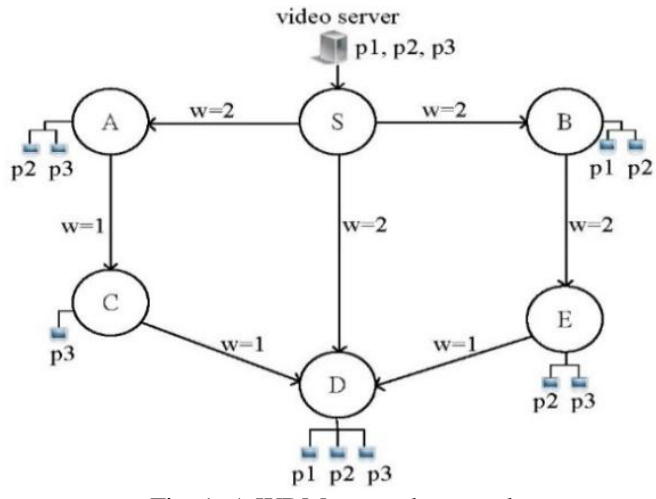

Fig. 1. A WDM network example.

\section{A. Constraints}

When a common link $l$ is shared by more than one multicast trees, the bandwidth constraint $C_{1}$ shown as follows must be satisfied:

$$
\sum_{0 \leq h \leq m-1} y_{h} \leq w(l), \text { where }\left\{\begin{array}{l}
y_{h}=1, \text { if } l \in T_{h} \\
y_{h}=0, \text { else }
\end{array}\right.
$$

Assume that wavelength $\lambda_{h 1}$ is assigned to the multicast tree $T_{i}$ and wavelength $\lambda_{h 2}$ is assigned to the multicast tree $T_{j}$, then the following wavelength assignment constraint $C_{2}$ must be satisfied:

$$
\left\{\begin{aligned}
\text { if }\left(\left\{l \mid l \in T_{i}\right\} \cap\left\{l \mid l \in T_{j}\right\} \neq \emptyset\right) \lambda_{h 1} & \neq \lambda_{h 2} \\
\text { else } & \lambda_{h 1}=\lambda_{h 2}
\end{aligned}\right.
$$

The constraint $C_{2}$ is used to make sure that two multicast trees must be allocated two different wavelengths unless they do not have any link in common.

Assume that a Boolean array $M C$ is given for setting multicast capability for each optical switch node in the graph $G$. When $M C[u]=$ false, node $u$ does not have multicast capability. As a result, $u$ can only be a leaf-node or an internal node with only one child node.A multicast capability constraint $C_{3}$ is hencedefined as follows:

If $M C[u]=$ false, the number of downstream nodes of

$$
u \leq 1 \text {, where } u \in T_{i}, 0 \leq i \leq m-1 \text {. }
$$

For a path $P$ from server $S$ to a destination $d$, the path delay of $P$ is defined to be $\sum_{l \in P} \rho(l)$. Assume the given delay bound is $\Delta$, each multicast tree $T_{i}$ must satisfy the following delay constraint $C_{4}$ :

$$
\operatorname{maximum}_{P \in T_{i}}\left\{\sum_{l \in P} \rho(l)\right\} \leq \Delta, 0 \leq i \leq m-1
$$

\section{B. Objective Function}

Before the objective function is presented for ourPDGRM problem proposed in this paper, several auxiliary data structures must be defined first. For each node $u \in V$, two vectors: demand $(Z)$, receive $(R)$, are created. These two vectors have the same size which is equal to the number ofstreams $m$. For the value of $Z_{u}[i]$ for node $u$, it represents the number of clients who send out requests for subscribing the $i t h$ video stream at node $u$.It is assumed that $Z_{s}$ is a zero vector at server node $S$.As for the vector $R$, it is a binary vector. If the ith stream is receivable in node $u, R_{u}[i]=1$. Otherwise, $R_{u}[i]=0$. For the server node $S, R_{S}[i]=1, \forall i \in$ $M$. The objective function of PDGRM problem can be then formulated as follows.

$$
\text { profit }=\operatorname{maximize}\left\{\sum_{T_{i} \in G T} \sum_{u \in T_{i}} Z_{u} \cdot R_{u}\right\}
$$

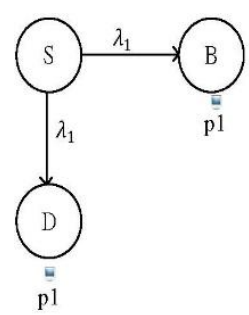

$T_{1}$

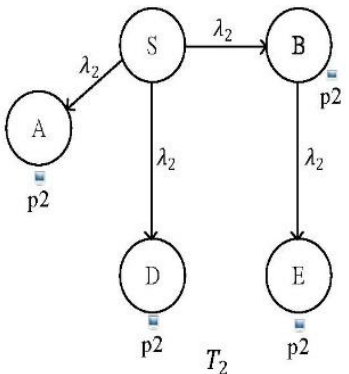

Fig. 2. Multicast group I.

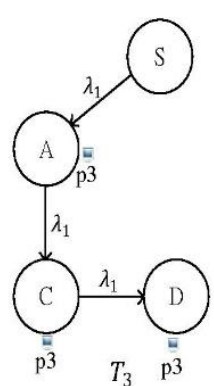

$T_{3} \mathrm{p}^{3}$

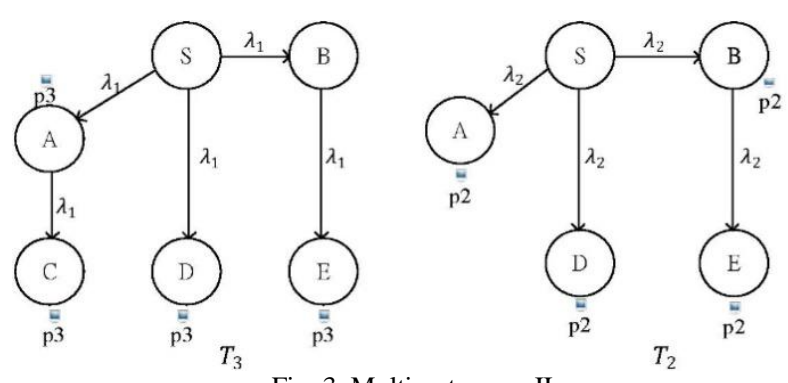

Fig. 3. Multicast group II.

\section{AN EXAMPLE}

For a WDM network given in Fig. 1, we may have two different multicast-tree construction results shown in Fig. 2 and Fig 3 respectively. In Fig. 2, three multicast streams are constructed and 9 clients are serviced, whereas there are only two multicast streams in Fig. 3 and only 8 clients can be serviced.

In order to determine the $R$ vectors of all multicast nodes, we also define a binary flow vector $F$ for each link $l$. For each $F_{l}[i]$, it is one if the ith stream is transmitted on the link $l$, and it is zero otherwise. In Fig. 4 , for example, $F_{S \rightarrow A}=$ $[0,1,1]$. It represents streams $p_{2}, p_{3}$ transmitted from $S$ to $A$. Therefore, $R_{A}=[0,1,1]$. On the other situation like node $D$, it has more than one incoming links, $R_{D}$ is the union of all $F$ 
vectors from the incoming links. After all the $F$ and $R$ vectors are determined, the multicast trees can be derived from them. The multicast trees shown in Fig. 2 are derived on the basis of $F$ and $R$ vectors computed in Fig. 4.

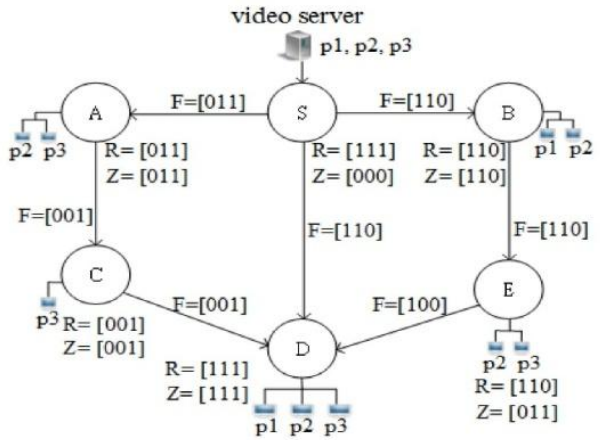

Fig. 4. Determination of receive vectors.

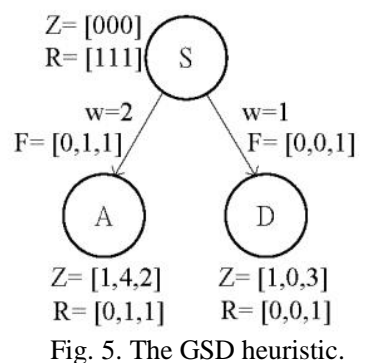

\section{GREEDY-BASED STREAM DistribUtion HeURISTIC}

Since the demand vector $Z_{u}[i]$ contains the number of clients subscribing the ithstream at node $u, \sum_{0 \leq i \leq m-1} Z_{u}[i]$ is the total number of clients subscribing streams at node $u$.Hence, starting from server node $S$, we assign $F$ vector to its direct child nodes so that the number of clients in each child node receiving the streams is maximized. This heuristic is named the greedy-based stream distribution (GSD) algorithm.

In Fig. 5, for example, $F_{S \rightarrow A}=[0,1,1]$ because the bandwidth of link $l_{S \rightarrow A}$ is 2 and $Z_{A} \cdot F_{S \rightarrow A}^{T}=6$ whichis the maximum among all possible assignments. The same method is also applied to link $l_{S \rightarrow D}$. This greedy-based distribution strategy is then recursively performed in breadth-first order. After all the flow and receive vectors are determined, the desired multicast trees are then extracted from the resulting network. These multicast trees must be pruned for multicast incapable non-leaf nodes which have more than one child node. At last, these resulting trees must be pruned again for recursively removing the unwanted leaf-nodes. An unwanted leaf-node is defined to be a leaf node which does not have any client subscribing the video streams.

\section{The Profit AND K-Shortest Paths Based Multicast TREES CONSTRUCTION ALGORITHM}

On the basis of k-shortest paths, a profit-oriented heuristic algorithm for constructing a group of delay-constrained multicast trees on a WDM network is developed in this section. This heuristic is referred as the profit and $\mathrm{k}$-shortest paths (PKSP) based algorithm, which is shown in Fig. 6. Theidea behindPKSP algorithm consists of the following two steps: multicast-tree construction phase and resource reallocating phase.

\section{A. Multicast-Tree Construction Phase}

In this phase, we construct a profit-oriented multicast tree $T_{h}$ for each stream without considering the wavelength assignment constraint $C_{2}$ shown in section II.

1) $T_{h}$ is computed based on the subgraph $G_{h}$ which is extracted from the given graph $G$. However, before $T_{h}$ is computed, a node weight $\Psi(u)$ for $u \in G_{h}$ and k-shortest paths from a destination $d \in D_{h}$ to each other node $u \in V_{h}, u \neq d$, must be computed determine first.

2) The $\Psi(u)$ is defined to the summation of the number of clients of all downstream nodes which can be reached from $u$. The details of this computation is described in Fig. 7.

3) For each subgraph $G_{h}$, the k-shortest paths from each destination $d_{i}$ to the other nodes are computed based on Yen's algorithm [10]. Only feasible paths whose delays are not greater than the given delay bound $\Delta$ are stored in a table named $\Gamma_{h}$.

4) $T_{h}$ is then computed by the profit-oriented multicast tree construction function described in Fig. 8. The construction is started from the server node. In Fig. 8, the destinations in set $D_{h}$ are sorted into a non-increasing sequence $\Pi_{h}$ based on node weight. Then, a multicast tree is constructed by iteratively adding the $\mathrm{k}$-shortest path which is from to the first destination of sorted sequence in $\Pi_{h}$. That is, the destination with the maximal node weight has the highest priority to be selected for addition.

5) When the shortest path $p_{u \rightarrow d}$ selected from $\Gamma_{h}$ is considered for addition to $T_{h}$, the following constraints must be satisfied:

- $u$ is a multicast-capable node or $u$ is a leaf node in $T_{h}$.

- $p_{u \rightarrow d}$ is not overlapped with $T_{h}$ except for $u$.

- After the path addition, the delay of the resulting tree should be no greater than the delay bound .

- If there are more than one path satisfied the above constraints, we select the path $p_{u \rightarrow d}$ with the largest $\Psi(u)$. This is a profit-oriented addition.

The destination $d$ is discarded when no path can satisfy the constraints (a)-(c).

The lines (9)-(18) in Fig. 6 are used for implementing the above procedure.

\section{B. Resource Reallocating Phase}

The multicast trees constructed in the first phase may contain the overloaded links. When the number of trees containing link $l$ is greater than bandwidth (the number of wavelength)pre-allocated to link $l, l$ is called an overloaded link. At line (19) in Fig. 6, the set $L$ is set to collect all the overloaded links.

1) For each overloaded link $l \in L$, we remove it from the best tree $T_{j}$ among all the trees containing $l$. At line (22) in Fig. $6, T_{j}$ with the smallest $\delta_{j}$ is selected where $\delta_{j}$ is the difference of the number of clients between $T_{j}$ and $T_{j}-l$. That is, we delete an overloaded link $l$ from a tree $T_{j}$ so that the loss of $\operatorname{gain}\left(T_{j}\right)$ is minimum. The $\operatorname{gain}\left(T_{j}\right)$ is defined to be the number of clients covered by $T_{j}$.

2) An uncovered destination set $Z_{h}$ is created for each tree 
$T_{h}, 0 \leq h \leq m-1$. We then try to add the uncovered destinations back to tree $T_{h}$ on the basis ofk-shortest paths stored in each $\Gamma_{h}, 0 \leq h \leq m-1$.

3) At lines (27)-(33) in Fig. 6, a set $\mathcal{P}$ is used to collect the feasible paths $p_{f, h}$ that can connect a destination $d_{i} \in \mathrm{Z}_{h}$ back to a node $u_{j} \in T_{h}$. Herewe assume there are $k$ shortest paths from node $u_{j}$ to $d_{i}$ and $0 \leq f \leq k$. A feasible path $p_{f, h} \in \mathcal{P}$ must satisfy two constraints: the delay of $\left(T_{h} \cup p_{f, h}\right) \leq \Delta$ and no overloaded link is generated for this path addition $\left(T_{h} \cup p_{f, h}\right)$.

4) If $\mathcal{P}$ is not empty, a path with the maximal path weight in $\mathcal{P}$ is selected for addition to the tree at lines (35)-(36) and then go back to line (26) for next iteration. If $\mathcal{P}$ is empty, algorithm stops.

Input: a directed graph $\mathrm{G}(\mathrm{V}, \mathrm{E})$, a server node $\mathrm{S}$ with $\mathrm{m}$ video streams, a destination set $\mathrm{D}$, a delay bound $\Delta$ and a MCarray.

1) Start at the server node $S \in G$, assign each node a level value in breadth-first order ;

2) Make $G(V, E)$ be acyclic direct graph by deleting backward links $l_{u \rightarrow v} \in G$, where level $(u) \geq \operatorname{level}(v)$;

3) For each $v \in V$, a demand array is defined as follows:

demand $[v][h]=\alpha$, is the number of clients for

the hth stream, $0 \leq h \leq m-1$;

4) For each destination $d \in D\{$

5) compute the shortest path $P_{d}$ for destination node $d$;

delete node $d$ if path delay of $P_{d}>\Delta$;

7) Recursively remove unwanted leaf-nodes;

8) $\}$

9) For each stream from to $m-1\{$

10) $D_{h}=\{v \mid$ demand $[v][h]>0, v \in V\}$;

11) $G_{h}\left(V_{h}, E_{h}\right)=$ Recursively remove unwanted

For each node $u \in G_{h}$ leaves in $G$ based on $D_{h}$

13) node weight $\Psi(u)=$ Compute_node_weight $\left(u, G_{h}\right)$;

14) For each destination $d_{i} \in D_{h}\{$

15) Create an array $\Gamma_{h, i}$ to store all the feasible $k$ - shortest pathsfrom node $u$ tod with path delay $\leq \Delta$ where $u \in V_{h}, u \neq d$

16) $T_{h}=$ Construct_multicast_tree $\left(D_{h}, G_{h}\right)$;

18) $\}$

19) Compute the link set $L, L=\left\{l_{u \rightarrow v} \mid\right.$ bandwidth of $l_{u \rightarrow v}<$ the number of $T_{h}$ containing $l_{u \rightarrow v}, 0 \leq h \leq m-1$;

20) For each link $l_{u \rightarrow v} \in L\{$

21) Let $Y=\left\{T_{j} \mid l_{u \rightarrow v} \in T_{j}\right\}$;

22) $\delta_{j}=\operatorname{gain}\left(T_{j}\right)-\operatorname{gain}\left(T_{j}-l_{u \rightarrow v}\right), T_{j} \in Y$;

23) Delete $l_{u \rightarrow v}$ from $T_{j}$ if $\delta_{j}$ is the smallest for $T_{j}$ among all the trees in ;

24) \}

25) Compute the uncovered destination set $Z_{h}$ for each tree $T_{h}$,

$\mathrm{Z}_{h}=\left\{d_{h, i} \mid d_{h, i}\right.$ is an uncovered

destination for $\left.T_{h}, 0 \leq i \leq \theta_{h}\right\}$,

$0 \leq h \leq m-1$;

26) $\mathcal{P}=\varnothing$

27) $\operatorname{For}(h=0 ; h<m ; h++)\{$

28) $\quad$ For $\left(i=0 ; i<\theta_{h} ; i++\right)\{$

// for each uncovered destination in tree $T_{h}$

For $\left(j=0 ; j<\left\|V_{h}\right\|-1 ; j++\right)\{$

//Assume there are $k$ shortest paths from node $u_{j}$ to $d_{i}$

30) For (each path $p_{f, h}$ in each entry $x_{h, i}[j] \in \Gamma_{h, i}$,

$0 \leq f \leq k)\{$

31) If (delay of $\left(T_{h} \cup p_{f, h}\right) \leq \Delta$ and $\left(T_{h} \cup p_{f, h}\right)$

32) $\left.\mathcal{P}=\mathcal{P} \cup p_{f, h} ;\right\}$

does not have any overloaded link)

33) $\}\}\}$

34) $\quad$ If $(\mathcal{P} \neq \emptyset)\{$

35) Let $p_{\max , h}=$ the path of the maximal weight in $\mathcal{P}$;

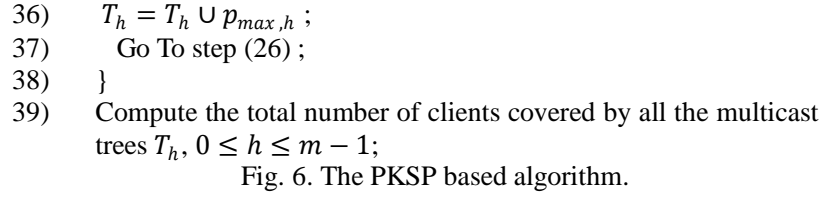

Integer Comput_node_weight(node $u$, Digraph $G$ ) \{

1) Let a queue $Q=\{u\}$;

2) $\operatorname{mark}[u]=$ visited; $\Psi(u)=0$

3) While $(\mathrm{Q} \neq \varnothing)\{$

4) $\quad v=\mathrm{Q}$.dequeue ()

5) $\Psi(u)+=\#$ of clients in node $v$

6) For each direct downstream node $w$ of $v\{$

7) $\quad$ If $(\operatorname{mark}[w]==$ unvisited $)\{$

8) $\operatorname{mark}[w]=$ visited

9) $Q=Q \cup\{w\}$;

10) $\}$

11) $\}$

12) \}

13) Return $\Psi(u)$

\}

Fig. 7. The node weight computation function

Tree Construct_multicast_tree(destination_set $D_{h}$, Digraph $\left.G_{h}\right)$

1) Let $T_{h}=\{\mathrm{S}\}, N^{\prime}=\mathrm{V}-T_{h}, D_{h}=\left\{d_{1}, \cdots, d_{f}\right\}$;

2) Let $\Pi_{h}=\left\{\right.$ sort destination nodes $d_{i} \in D_{h}$ into a non increasing sequence based on $\left.\Psi\left(d_{i}\right)\right\}$;

3) While $\left(\Pi_{h} \neq \emptyset\right)\{$

4) $\quad d=$ the first element of $\Pi_{h} ; \Pi_{h}=\Pi_{h}-\{d\}$;

5) From Table $\Gamma_{h}(d)$, select a path $p_{u \rightarrow d}$ which can

connect $d$ back to a node $u \in T_{h}$, such thatthe

following constraints are satisfied:

(a) $u$ is a multicast-capable node or $u$ is a leaf node in $T_{h}$

(b) $u=T_{h} \cap p_{u \rightarrow d}$;

(c) The longest path delay of $\left(T_{h} \cup p\right) \leq \Delta$

(d) Node weight $\Psi(u)$ is the maximum;

6) $T_{h}=T_{h} \cup p$;

7) If no path $p$ can satisfy the above constraints

(a) (c), discard node $d$;

8) \}

9) Return $T_{h}$

Fig. 8. The profit-oriented multicast tree construction. function.

\section{EXPERIMENTAL RESULTS}

In this section, a number of experiments are conducted for comparing the performances forPKSP, GSD, Member-only and Member-first heuristic algorithms in terms of network size, number of streams, delay bound and multicast capability. The Member-only and Member-first heuristics proposed by Zhang [5] are two well-known multicast forest construction algorithms. In this study, these two methods are modified to determine a multicast tree only and ignore those uncovered destinations, since there is only one wavelength available for each multicast stream.

All programs implementing the aforementioned heuristics are written by Java. The experiments are carried out in a desktop PC running Windows 7 on Intel i7-3770 CPU and 8GB RAM. All data reported in this study is an average value on 100 runs.

As for the simulation settings are as follows: network size is $50-100$ nodes, multicast ratio is $10 \%-80 \%$, the number of streams $(m)$ is $10-20$, and the number of wavelengths on a link is a random integer no greater than $m$, and the number of clients at each node is no greater than 5 . The delay of each link is a random integer between 1 and 5 , so the delay bound of a multicast tree is set to be $20-50$ which is dependent on network size. 
In literature [11], it reported that in general no more than $50 \%$ nodes need to have multicast capability in order to have close performance of a WDM network with full light splitting capability. In this study, hence, the ratio of multicast-capable $(m c)$ nodes is set to be $0.2-0.5$ to observe the performance difference for the proposed heuristics.Each node is randomly selected to be a multicast-capable node.

\section{A. Random Directed Graph Generation}

For the experiments conducted in this section, we have a procedure in the algorithm for generating random directed graphs. First, we generate a random undirected graph based on the following equation:

$$
\operatorname{Prob}\{\{u, v\}\}=\beta \exp \frac{-\operatorname{dist}(u, v)}{L_{\max } \alpha}
$$

where Prob is the probability for making a link from $u$ to $v$, $\operatorname{dist}(u, v)$ is the Euclidean distance from $u$ to $v, L_{\max }$ is the maximum distance of any two nodes in the graph, $\alpha$ and $\beta$ are two positive and less than one numbers [12]. In this study, we set $\alpha=0.5$ and $\beta=0.5$. The undirected graph is then converted to an acyclic directed graph where the maximum degree of a node is no greater 4 .

\section{B. Performance Comparisons}

The performance measured for all heuristics is computed based on the profit defined in (5) in Section II. In fact, the value of profit is the total number of serviced clients supported by all the multicast trees.

In Fig. 9 and 10, 100-node and 50-node networks are used for comparisons when $m c=0.5$. In general, the GSD method is slightly better than Member-only and Member-first heuristics. However, our PKSP method can consistently outperform the other three approaches in terms of various multicast ratios. This superiority increases as the number of destinations increases. In particular, the profit values of GSD are around $70 \%-78 \%$ of the profit values of PKSP for 100-node networks.

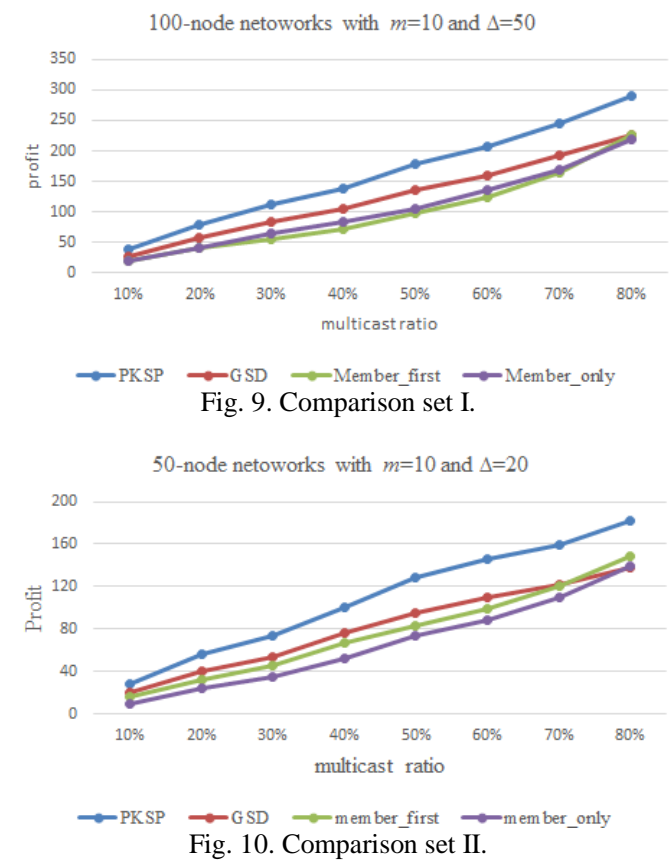

The second set of comparisons is done for different number of streams. In Fig. 9 and 11, the number of streams are set to be 10 and 20 respectively. Obviously, the performance trend is similar for $m=10$ and $m=20$. The PKSP method still gives us the best profit value.

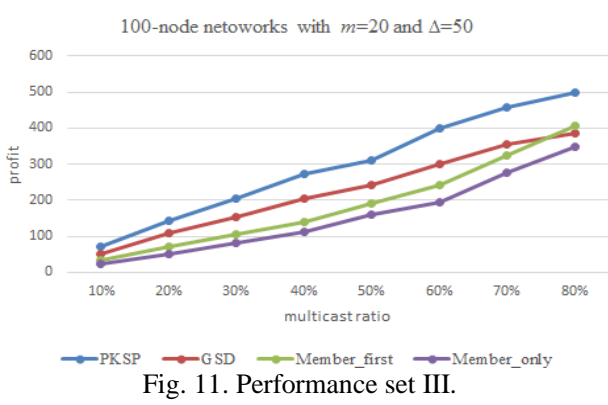

The third set of comparisons is done for different delay bound. In Fig. 9 and 12, the delay bounds are set to 50 and 20 respectively. When $\Delta=20$, the profit valuesof GSD are around $64 \%-75 \%$ ofthe profitvaluesof PKSP for 100-node networks.Compared to the case with $\Delta=50$, it shows that the PKSP algorithm is more suitable for real-time applications with strict delay bound.

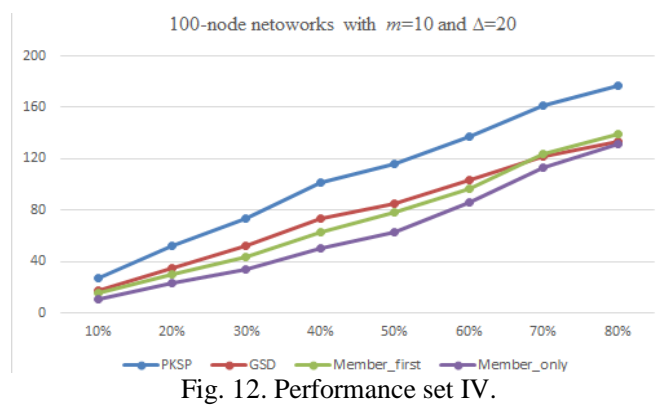

The first three sets of performance comparisons are done for the same ratio of multicast-capable nodes, $m c=0.5$. In this set of comparisons, we set $m c=0.2$ for the data shown in Fig. 13. Although the PKSP method still has the best performance in Fig. 13, the performance trend is different it in shown in Fig. 9. The main difference is that when the multicast ratio approaches $80 \%$, the superiority of ourPKSP method becomes non-obvious. It is because that for small $m c$ ratios and high multicast ratios the multicast trees are formed mainly by the non-overlapping shortest paths from server node to each destination $d$. Therefore, all heuristics devised on the basis of the k-shortest paths intends to give us the similar performance.

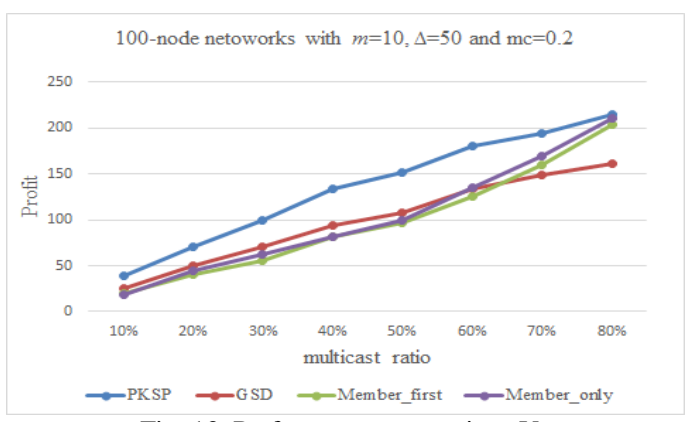

Fig. 13. Performance comparison V.

\section{CONCLUSION}

In this paper, a resource constrained problem called 
PDGRM is proposed. It concerned about how to multicast a number of streams simultaneously to a number of clients in a given WDM network with sparse light spitting, where the number of wavelengths on each link is given in advance.

Our goal is to construct a number of multicast trees with the maximal serviced clients for all required streams, and also satisfying the following constraints: multicast capability, bandwidth constraint, delay bound. Two heuristic algorithms: PKSPand GSD, are proposed for the problem. According to our experimental results, they show that the PKSP method can consistently outperform GSDmethod and the other two previously published methods in terms of network size, the number of video streams, delay bound and the number of multicast-capable nodes.

\section{REFERENCES}

[1] J. He, S. H. Chan, and H. K. Tsang, "Multicasting in WDM networks," IEEE Communication Surveys, vol. 4, no. 1, pp. 2-20, 2009.

[2] A. Ding and G.-S. Poo, "A survey of optical over WDM networks," Computer Communications, vol. 26, pp. 193-200, 2003.

[3] S. Barat et al., "A light-forest approach for QoS multicasting in WDM networks," in Proc. 7th IEEE Conference on Industrial and Information Systems, 2012, pp. 1-6.

[4] A. Hamad, T. Wu, A. E. Kamal, and A. K. Somani, "On multicasting in wavelength-routing mesh networks," Computer Networks, vol. 50, pp. 3105-3164, 2006.

[5] X. Zhang, J. Y. Wei, and C. Qiao, "Constrained multicast routing in WDM networks with sparse light splitting," Journal of Lightwave Technology, vol. 18, no. 12, pp. 1917-1927, 2000.

[6] X.-H. Jia, D.-Z. Du, and X. D. Hu, "Integrated algorithms for delay bounded multicast routing and wavelength assignment in all optical networks," Computer Communications, vol. 24, pp. 1390-1399, 2001

[7] J. He, S. H. Chan, and H. K. Tsang, "Routing and wavelength assignment for WDM multicast networks," in Proc. IEEE Global Telecommunications Conference, 2001, pp. 1536-1540.

[8] C.-F. Wang, B.-R. Lai, and R.-H. Jan, "Optimum multicast of multimedia streams," Computers \& Operations Research, vol. 26, pp. 461-480, 1999.

[9] Y. Cao and O. Yu, "On the study of group multicast in WDM networks," in Proc. IEEE Conference on Communications, 2005, vol. 3, pp. 1625-1630.

[10] J. Y. Yen, "Finding the K shortest loopless paths in a network," Management Science, vol. 17, no. 11, pp. 712-716, 1971.

[11] R. Malli, X. Zhang, and C. Qiao, "Benefits of multicasting in all-optical networks," in Proc. the SPIE, 1998,vol. 3531, pp. 209-220.

[12] B. M. Waxman, "Routing of multipoint connections," IEEE Journal of Select Areas Communications, vol. 6, pp. 1617-1622, 1988.

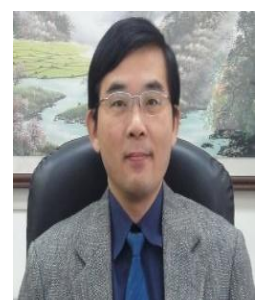

Wen-Lin Yang received his Ph.D. and M.S. in computer science from Pennsylvania State University and State University of New York, in 1993 and 1988, respectively. Currently, he serves as the dean of College of Science and Engineering as well as a professor in the Department of Computer Science and Information Engineering at National University of Tainan, Taiwan. His primary research interests are optical networks, routing protocols, wireless networks, and distributed computing.

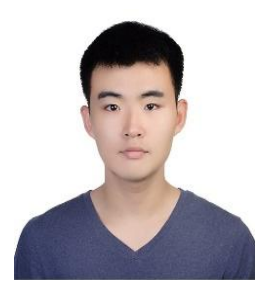

Chun-Tao Yang is currently a senior student pursuing a bachelor's degree in electrical and computer Engineering at National Chiao Tung University, Hsinchu, Taiwan. His primary research interests include network programming and robotics control and programming.

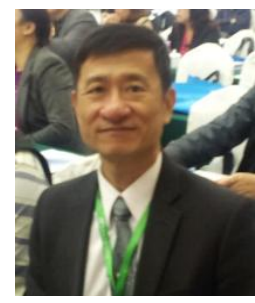

management.
Yu-Chung Huang received his M.S. in chemica engineering from National Cheng Kung University of Taiwan in 1983. He is a PhD student in electrica engineering at National University of Tainan, Taiwan. He also works as a consultant of Wasion group, which is a leading energy measurement instrument, system and service supplier in China. His primary research interests include machine learning, communication systems, and resources 